\title{
Maternal-fetal outcomes from a pregnant 28-I weeks woman under four months coma after multi- traumatic brain injury: a case report
}

\begin{abstract}
In Feb 2015, a pregnant $28^{-1}$ weeks woman who was involved in a catastrophic motor vehicle accident with severe brain injuries and systemic multiple fractures was admitted to our emergency room. The patient accepted emergent cesarean section to save the fetus and the subsequent life-sustaining multi disciplinary treatment, and finally recovered from a 4-month coma. This case is presented to explore the delivery outcomes and craniectomy in pregnant women with catastrophic brain injury, which aims to enable clinicians to better appreciate the history and current issues involving advance directives for pregnant women, maternal versus fetal interests, and the impact of fetal viability on medical decision making in pregnant women with catastrophic brain injury and to encourage the use of persistent multi disciplinary treatment to save lives..
\end{abstract}

Keywords: traumatic brain injury, pregnant woman, cesarean section
Special Issue - 2018

\author{
Xue-Tang Mo, Cui-Lan Li, Shi-Yan Tang \\ Department of Obstetrics and Gynecology, Guangzhou Medical \\ University, China
}

Correspondence: Cui-Lan Li, Department of Obstetrics and Gynecology, The Third Affiliated Hospital of Guangzhou Medical University \& Key Laboratory for Major Obstetric Diseases of Guangdong Province \& Key Laboratory of Reproduction and Genetics of Guangdong Higher Education Institutes, Guangzhou 5I0I45, P. R. China, 5516 Glenwood Rd, Bethesda, MD, 208I7, USA, Tel + I (9I3) 406 4647, Fax +86-20-8I292949,

Email ninacuilanli@gmail.com

Received: January 14, 2018 | Published: November 30, 2018

\section{Introduction}

Head and neck injuries, respiratory failure, and hypovolemic shock are the three most common causes of trauma related to maternal mortality in pregnancy. ${ }^{1-2}$ Trauma during pregnancy, including brain and neurological injury, is one of the leading causes of incidental maternal mortality and morbidity and complicates $6-7 \%$ of all pregnancies. $^{3-4}$ Traumatic brain injuries and neurological injuries during pregnancy, although less common than abdominal trauma, have greater risk in terms of morbidity and mortality. ${ }^{5,6}$ Moreover, two lives are in danger, leading todistinct diagnostic and therapeutic challenge. ${ }^{3}$ Common etiologies for trauma during pregnancy are motor vehicle accidents $(54.6 \%)$, falls from heights $(21.8 \%)$, violent assaults, and burns. ${ }^{7}$ A multidisciplinary approach involving neurosurgeons, anesthesiologists and obstetricians is mandatory for the successful management of such patients. ${ }^{8-10}$ This management consists of hemodynamic stabilization of the mother, systematic neurological assessment, and a secondary survey considering the evaluation of the fetus and delivery outcomes, followed by persistent life-sustaining treatment, craniectomy, and external fixation for fractures.

We present the case of a pregnant woman combined with severe traumatic brain injury, multiple skull fractures, subdural hemorrhage, subarachnoid hemorrhage, left tibia and fibula fractures, right crus contusion, and right occipital scalp contusion at her $27^{+6}$ weeks' gestation. The patient received treatment through a multidisciplinary approach involving a neurosurgeon, anesthesiologist, orthopedist, and obstetrician. The patient provided written consent for her data to be published. Issues such as screening of the mother, feto-maternal monitoring, physiological changes during pregnancy, intraoperative concerns, and timing of the termination of pregnancy are discussed alongside a review of pertinent literature.

\section{Case report}

The patient, a 27 -year-old woman at $28^{-1}$ weeks' gestation, was involved in a catastrophic motor vehicle accident with resultant severe traumatic brain injury and multiple systemic skull and lower limb fractures. Her Glasgow Coma Score (GCS) was 4 (E1V1M2) at the accident scene, and both of her pupils were dilated and nonreactive to light. She was taken to the local hospital as soon as possible and immediately received intravenous administration of furosemide, mannitol, and Green's solution; her fractured left limb was immobilized with an external splint. She was transferred to our high-risk pregnancy unit 3 hours after the accident. This was her first pregnancy, which had been uncomplicated until the accident. In our emergency room, she was found to have GCS 4 (E1V2M2), heart rate 71 beats/min, blood pressure (BP) $120 / 60 \mathrm{mmHg}$, and respiratory rate 18 breaths $/ \mathrm{min}$, and her pupils were dilated (left: $4 \mathrm{~mm}$; right: 5 $\mathrm{mm}$ ) and non-reactive to light. The patient was immediately intubated and assisted with mechanical ventilation as she remained comatose. Injuriesincludeda lacerated wound over the right temporal region and right lateral crus contusion, tears and extravasation; the left leg was immobilized with a splint, but local skin bruising was noted along with a palpable sense of bone movement.

A computed tomography (CT) scan of the head revealed right fronto-temporo-parietal acute subdural hemorrhage and subarachnoid hemorrhage causing midline shift; a depressed right parietal bone fracture with intracranial pneumatosis and merged right parietal scalp hematoma was detected; the upper apex, proximal wall, and right of the frontal sinus were fractured; hematocele were found in the bilateral ethmoid and sphenoid sinus; the left mastoid bone and the intact canal were fractured; hematocele were found in the left mastoid, tympanum, and external auditory canal; and effusion was seen in the right mastoid, which was suspected to be fractured. A multidisciplinary team consisting of a neurosurgeon, obstetrician, orthopedist, and anesthesiologist thoroughly discussed the situation with the patient's family, who requested that every possible intervention be attempted in the hope of improving the patient and fetus survival chances. The neurosurgeon concluded that 
there were no indications for surgical intervention; thus, the patient was first treated by dehydration to reduce intracranial pressure and then received ventilation with supportive treatment (life-sustaining treatment). Theorthopedist treated the left tibia and fibula fracture and the right crus contusion by immobilizing the patient's left leg with a splint that held the bones in position and immobilizing the joints above and below the fracture. Debridement and suture were performed for the lacerations on her right leg. Osteofascial compartment syndrome was ruled out by observation of the initial post-fracture edema, and the swelling decreased. At the same time, the patient was given an antibioticand injected with Human Tetanus Immunoglobulin (TIB).

The hemoglobin $(\mathrm{Hb})$ concentration was $7.1 \mathrm{~g} / \mathrm{dL}$, and the Ddimer was $11,212 \mathrm{ng} / \mathrm{ml}$; the other laboratory values were normal. Her body temperature was $38.8^{\circ} \mathrm{C}$. An obstetric examination utilizing ultrasonography revealed primary placental abruption, a fetal weight of approximately $1000 \mathrm{~g}$, a fetal heart rate of 95-100beats/min and repeated late deceleration, which was diagnosed as fetal bradycardia, suggesting fetal distress as per our experience. Because placental abruption was accompanied by signs of fetal distress, continuing the pregnancy would have risked the development of complications, such as stillbirth, coagulation disorders, disseminated intravascular coagulation (DIC), and multiple organ dysfunction syndromes (MODS). The patient had no obvious uterine contractions, vaginal bleeding, or signs of the impending rupture of membranes. Vaginal examination showed that the cervix was centered and closed, the regression rate of the cervical canal was $30 \%$, and the presentation of the fetus was floating. After discussing the risks and benefits of the different management options among our multidisciplinary team and the patient's family, it was decided that a cesarean delivery would be performed expeditiously, and the patient received an injection of dexamethasone to promoting fetal lung maturation.

During the operating, the patient was placed in a $30^{\circ}$ head-up position with a $15^{\circ}$ left lateral tilt. Monitoring included invasive BP, electrocardiography, pulse oximetry, and capnography. Delivery by cesarean section under general anesthesia was performed. A live, $1180 \mathrm{~g}$ male infant was delivered after 6 min of induction of anesthesia with Apgar scores of 7, 7, and 10 at 1, 5, and 10min, respectively. At delivery, the baby was resuscitated and transferred to the neonatal intensive care unit (NICU). After delivery, oxytocin was administered to the patient (10 U administered slowly i.v. over $5 \mathrm{~min}$, followed by the infusion of $20 \mathrm{U}$ in $500 \mathrm{ml}$ of normal saline over the next 4 h). The placenta was delivered intact. Intraoperative bleeding was approximately $200 \mathrm{ml}$. The mother was transferred to the intensive care unit (ICU), intubated and placed on mechanical ventilation after the cesarean section. On Post-Operative Day 1 (POD \#1), the patient was found to have high intracranial pressure (ICP, $180 \mathrm{~mm}$ $\mathrm{H}_{2} \mathrm{O}$ ) and was administered i.v. furosemide and mannitol. The patient remained in coma, but her orbital dynamic response was positive. At POD \#2, the patient responded to painful stimuli. She was febrile, and a sputum culture revealed the presence of gram-positive cocci. A tracheotomy was performed to assist respiration, and the patient was injected with imipenem and cilastatin sodium. A chest X-ray showed infection in the right pneumothorax, and a chest tube was inserted forclosed drainage. At POD \#4, her respiration improved, and her $\mathrm{SpO} 2$ was maintainedat $90 \%-95 \%$ without mechanical assistance. As mechanical ventilation was no longer required and the patient was afebrile, even she remained in a unconscious coma, she was transferred to the ward. However, after the transfer, repeated sputum cultures were positive for Acinetobacter baumannii, and teicoplanin was administered to combat the infection.
After 2 months, the patient was still in coma and hospitalized; a CT scan indicated that, compared with the initial CT scan, the patient's brain edema was in regression. She was diagnosed as sub-coma and her GCS was evaluated as 12 (E4V2M6). Her BP was 120/60mmHg, her respiratory rate was 20 breaths/min, and her pupils were normal (left: $3 \mathrm{~mm}$; right: $2.5 \mathrm{~mm}$ ) and sensitive to light. Her limb muscular tension and muscle strength were normal; physiological reflexes existed, and pathological reflexes were not induced. The neurosurgeon reported that the patient was stable, and her CT scan suggested an epidural hematoma and an edema that had indications for surgical intervention. In addition to a craniotomy (June 2015), right ventricle peritoneal shunt, evacuation of the epidural hematoma, and depressed skull fracture plasty were performed under general anesthesia. The orthopedist found that the patient needed external fixation of the left tibia and fibula, and internal fixation with plate and screw fixation and bone graftingwere performed under epidural anesthesia. After 4month coma and hospitalization (on June 26, 2015), the patient was gradually conscious and hemodynamically stable, and her laboratory values were normal. She was making good progress at discharge. Her baby did not require mechanical ventilation but was strictly observed in the NICU for 55 days and eventually discharged with a body weight of $6400 \mathrm{~g}$.

\section{Discussion}

Trauma in pregnancy is a leading non-obstetric cause of maternal death, and motor vehicle accidents are the most common cause of such trauma. ${ }^{3,11}$ Pregnant trauma victims present a distinctive spectrum of challenges for multidisciplinary healthcare teams. It is accepted practice that early aggressive maternal stabilization must be the priority in the management of a pregnant patient who has sustained major trauma because effective maternal resuscitation also provides fetal resuscitation. ${ }^{12}$ Pregnant patients with severe head injuries have an increased risk of fetal death. ${ }^{3,12}$ The early recognition of fetal compromise is therefore important in this group of patients. Up to $60 \%$ of fetal deaths result from delayed recognition of fetal distress and delayed cesarean section. ${ }^{13}$ All pregnant trauma patients should have their fetus monitored from the time of injury, and such monitoring should be continued for an appropriate interval until the danger has passed. ${ }^{12}$ Our pregnant 28-1 week's woman received prompt cesarean delivery within 48 hours after the traumatic brain injury as the ultrasonography and fetal monitor indicated a primary placental abruption and suspected fetal distress. As our hospital was experienced in treating preterm infants, our multidisciplinary healthcare team decided to give birth after promoting fetal lung maturation which would improve the prospects of survival; and the baby was transferred NICU for further management. Although the mother's condition was unstable, emergency cesarean delivery was necessary and vital to save lives when mother and the fetal lives at risk. If the mother's condition is stable, the status of the fetus and the extent of uterine injury determine further management. A potentially viable fetus that shows no signs of distress should be monitored by external ultrasonography or frequent fetal heart rate monitoring and a selective cesarean section was considered.

Placental abruption complicates $1-5 \%$ of minor injuries and $20-50 \%$ of major injuries. ${ }^{3,14,15}$ Excluding maternal death, placental abruption is the most frequent cause of maternal death after trauma. Fetal death resulting from injuries to the obstetric patient is most commonly associated with placental abruption. ${ }^{3,14}$ In our case, the patient had no obvious uterine contractions, vaginal fluid, or bleeding after injury. However, ultrasonography revealed primary placental 
abruption, a fetal heart rate of $100-112$ beats/min (considered as fetal bradycardia), and suspected fetal distress. We first suggested a termination of the pregnancy and then performed the cesarean section delivery of the fetus with the consent the patient's family. The fetus depends on its mother for its oxygen requirements; thus, an uninterrupted supply of oxygenated blood must be provided to the fetus at all times. Therefore, the timely management of hypotension is required in all females of child-bearing age, irrespective of whether a pregnancy is known to be present preoperatively. ${ }^{4,9}$ When a viable fetus shows signs of distress, a cesarean delivery must be performed expeditiously. Our patient performed a low diastolic blood pressure, the cesarean delivery would relive cardiac after load and improve the effective circulation blood volume supplement.

However, if the fetus was suspected non-viable, in utero conservative management may optimize maternal oxygenation and circulation. As physiological, hormonal, hemodynamic, and anatomical changes are associated with pregnancy, there were not a standard neurosurgical and orthopedic practices for pregnant women with traumatic brain injuries. ${ }^{3}$ No class 1 or class 2 evidence exists to guide the clinical management of such patients, and practice experience was mainly according to case reports. ${ }^{3}$ In this case, the patient accepted emergent cesarean delivery and the subsequent life-sustaining from multidisciplinary treatment and her family, and finally recovered from a 4-month coma. Therefore, close monitoring, constant discussion and support between the multidisciplinary healthcare team and the patient's family are essential.

In our case, the successful outcome for both the mother and child was facilitated by a multidisciplinary team approach. There was an obvious need to evacuate the craniocerebral injuries and systemic multiple fractures, yet immediate cesarean section were required to deliver the compromised fetus. In such cases, if the patient has stable vital signs, further treatment for craniocerebral injuries and multiple bone fractures should be applied after delivery of the fetus. For second trimester pregnant women with traumatic brain injury, positive and timely obstetrics surgical treatment may lead a hopeful and favorable prognosis. It is hoped that the present article encourages the use of persistent multidisciplinary treatment to save lives.

\section{Acknowledgments}

None.

\section{Conflict of interest}

The author declares no conflict of interest.

\section{References}

1. Baker BW, Trauma. In: Chestnut DH, editor. Obstetric Anesthesia, Principles and Practice. USA: St Louis Mosby; 1999. p. 1041-1050.

2. Rudra A, Ray A, Chatterjee S, et al. Trauma in Pregnancy. Indian J Anaesth. 2007;51(2):100-105.

3. Pankaj Dawar, AradhanaKalra MS, Deepak Agrawal, et al. Decompressive craniectomy in term pregnancy with combined caesarean section for traumatic brain injury. The Indian Journal of Neurotrauma 2014;11(12):162-165.

4. Shah AJ, Kilcline BA. Trauma in pregnancy. Emerg Med Clin North Am. 2003;21(3):615-629.

5. Mani Charan Satapathy, Sudhansu Sekhar Mishra, Srikanta Das, et al Emergency management strategy for pregnant head trauma victims Case reports and review of literatures. The Indian Journal of Neurotrauma. 2014;11(01):45-48.

6. Christopher M Burkle, Jennifer Tessmer-Tuck, Eelco F Wijdicks. Medical, legal, and ethical challenges associated with pregnancy and catastrophic brain injury. International Journal of Gynecology and Obstetrics. 2015;129(3):276-280.

7. Connolly AM, Katz VL, Bash KL, et al. Trauma and pregnancy. Am J Perinatol. 1997;14(6):331-335.

8. Reddy SV, Shaik NA, Gualala K. Trauma during pregnancy. J Obstet Anaesth Crit Care. 2012;2(1):3-9.

9. Rabia Qaiser, Peter Black. Neurosurgery in Pregnancy. Seminars in Neurology. 2007;27(5):476-481.

10. Mohamed Mohamed Tawfik, Basma Abed Badran, Ahmed Amin Eisa, et al. Simultaneous cesarean delivery and craniotomy in a term pregnant patient with traumatic brain injury. Saudi J Anaesth. 2015;9(2):207210 .

11. Aitokallio-Tallberg A, Halmesmaki E. Motor vehicle accident during the second or third trimester of pregnancy. Acta ObstetGynecol Scand. 1997;76(4):313-317.

12. Goldman SM, Wagner LK. Radiologic ABCs of maternal and fetal survival after trauma: when minutes may count. Radiographics. 1999;19(5):1349-1357.

13. Morris JA, Rosenbower TJ, Jurkovich GJ, et al. Infant survival after cesarean section for trauma. Ann Surg. 1996;223(5):481-491.

14. Schiff MA, Holt VL. The injury severity score in pregnant trauma patients: predicting placental abruption and fetal death. J Trauma. 2002;53(5):946-949.

15. Kettel LM, Branch DW, Scott JR. Occult placental abruption after maternal trauma. Obstet Gynecol. 1988;71(3 pt 2):449-453. 